\title{
An update on the treatment of rosacea
}

\section{Alexis Lara Rivero \\ Clinical research fellow \\ St George Specialist Centre \\ Sydney \\ Margot Whitfeld \\ Visiting dermatologist \\ St Vincent's Hospital \\ Sydney \\ Senior lecturer \\ UNSW Sydney}

\section{Keywords}

flushing, rosacea

Aust Prescr 2018;41:20-4 https://doi.org/10.18773/ austprescr.2018.004

\section{SUMMARY}

Rosacea is a common inflammatory skin disorder that can seriously impair quality of life.

Treatment starts with general measures which include gentle skin cleansing, photoprotection and avoidance of exacerbating factors such as changes in temperature, ultraviolet light, stress, alcohol and some foods.

For patients with the erythematotelangiectatic form, specific topical treatments include metronidazole, azelaic acid, and brimonidine as monotherapy or in combination. Laser therapies may also be beneficial.

For the papulopustular form, consider a combination of topical therapies and oral antibiotics. Antibiotics are primarily used for their anti-inflammatory effects.

For severe or refractory forms, referral to a dermatologist should be considered. Additional treatment options may include oral isotretinoin, laser therapies or surgery.

Patients should be checked after the first 6-8 weeks of treatment to assess effectiveness and potential adverse effects.

\section{Introduction}

Rosacea is a common chronic relapsing inflammatory skin condition which mostly affects the central face, with women being more affected than men. ${ }^{1}$ The pathophysiology is not completely understood, but dysregulation of the immune system, as well as changes in the nervous and the vascular system have been identified. Microbes that are part of the normal skin flora, and specifically in the pilo-sebaceous unit - including Demodex mites and Staphylococcus epidermidis - may also play a role as triggers of rosacea. ${ }^{2,3}$

Symptoms are initially transient. This is followed by persistent erythema due to repeated vasodilation, then telangiectasia and skin inflammation in the form of papules, pustules, lymphoedema and fibrosis. ${ }^{2,4}$

Rosacea can seriously affect a patient's quality of life. This should prompt clinicians to diagnose it early and start treatment.

\section{Diagnosis}

The diagnosis of rosacea is usually made on history and clinical features. If it is not clear, differential diagnoses must be considered and ruled out (see Box). ${ }^{1,5,6}$

\section{Clinical manifestations}

The presence of at least one of the following primary features indicates rosacea:

- flushing (transient redness)

- non-transient redness
- papules

- pustules

- telangiectases.

In addition, at least one of the secondary features of burning or stinging, a dry appearance, plaque formation, oedema, central facial location, ocular manifestations and phymatous changes are considered enough to make the diagnosis accurately in most cases. Rosacea usually follows a pre-rosacea stage that involves flushing only.

\section{Box Differential diagnoses of rosacea}

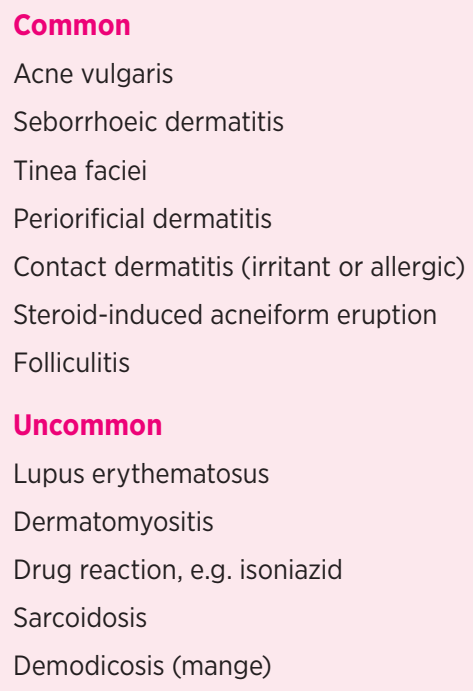

Source: References 1, 4, 6 
Rosacea can be classified into four subtypes:

erythematotelangiectatic, papulopustular, phymatous and ocular. ${ }^{1,5}$

\section{Erythematotelangiectatic rosacea}

Erythematotelangiectatic rosacea is characterised by flushing and persistent central facial erythema. Redness may also involve the peripheral face, ears, neck and upper chest, but periocular skin is typically spared. Telangiectases are also common, but are not required for the diagnosis (Fig. 1).

\section{Papulopustular rosacea}

Papulopustular rosacea subtype includes patients who develop papules or pustules in a central facial distribution. In severe cases, these episodes of inflammation can lead to chronic facial oedema (Figs 2 and 3).

\section{Fig. 1 Erythematotelangiectatic rosacea}

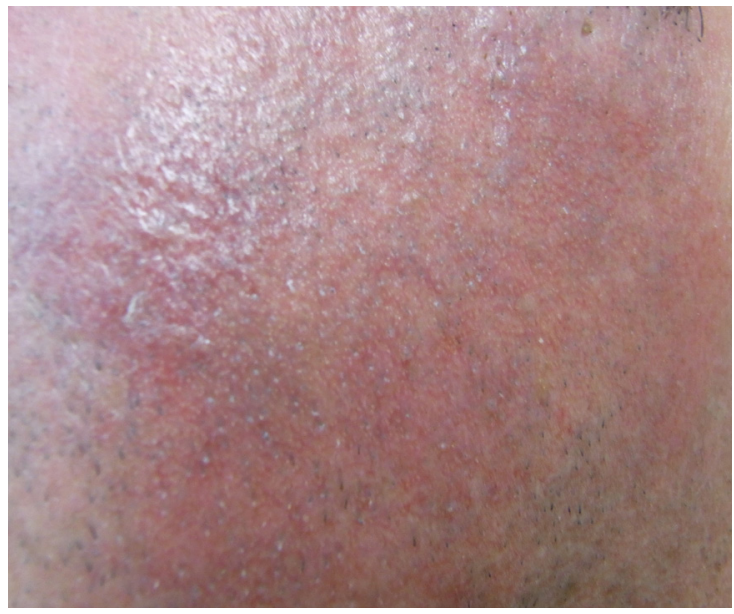

\section{Fig. 2 Papulopustular rosacea}

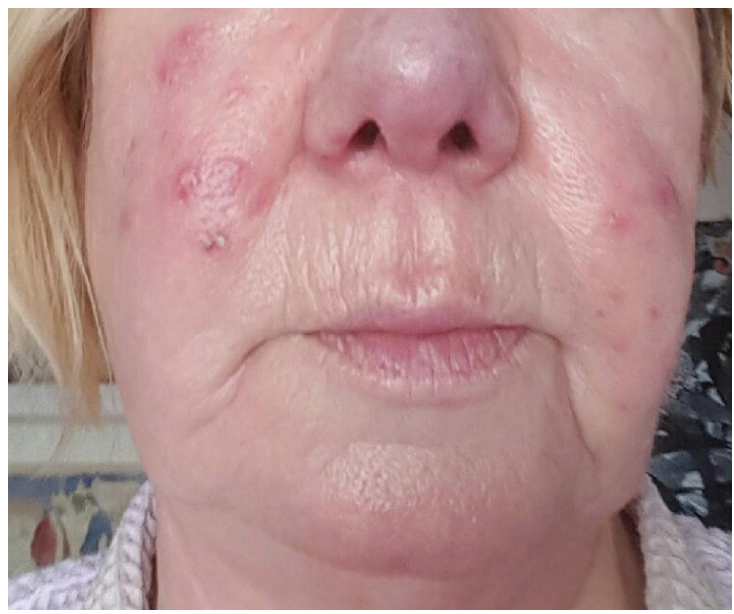

\section{Phymatous rosacea}

Phymatous rosacea is characterised by thickened skin with enlarged pores and irregular surface nodularities. These changes are most commonly found on the nose (rhinophyma), but can occur on the ears, chin and forehead. This subtype is more common in men than women (Fig. 4).

\section{Ocular rosacea}

Ocular rosacea is characterised by a watery or bloodshot appearance of the eyes, foreign body sensation, burning or stinging. Blepharitis, conjunctivitis, dryness, itching, light sensitivity, blurred vision and telangiectasia of the conjunctiva or eyelids also occur. Chalazia and styes are more common in ocular rosacea than other forms. Because there is no specific test, the diagnosis relies on the physician's

\section{Fig. 3 Papules and pustules - close-up}

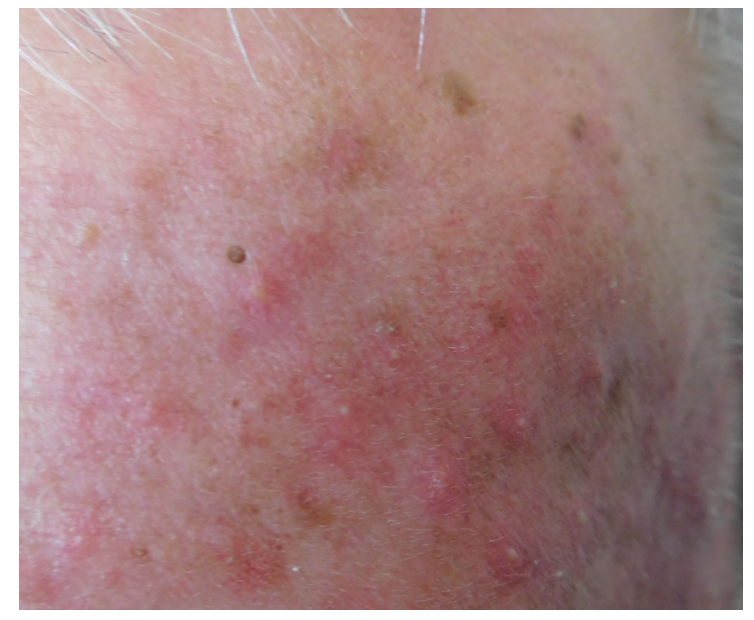

\section{Fig. 4 Rhinophyma}

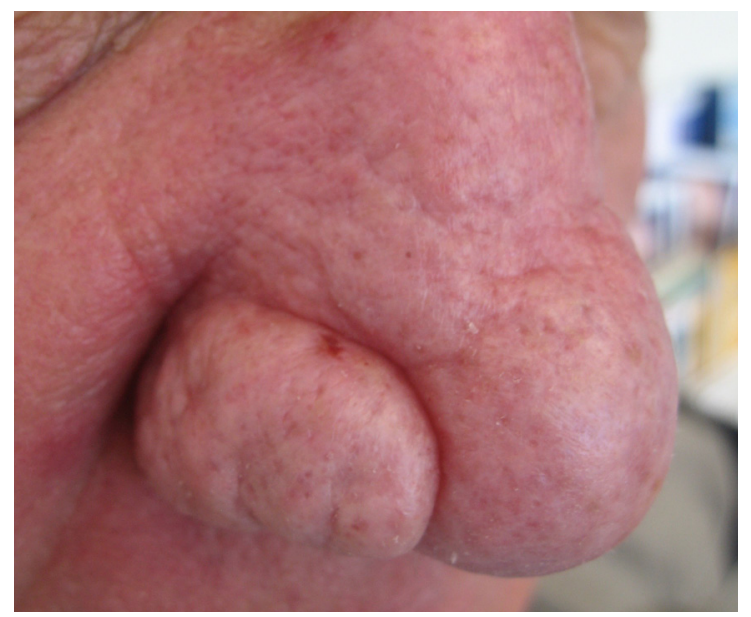


clinical judgment. Ocular involvement is estimated to occur in $6-50 \%$ of patients with cutaneous rosacea, and can occur with or without a diagnosis of cutaneous rosacea.?

\section{Additional tests}

If the diagnosis cannot be made clinically, other tests may be necessary. These include skin swabs and scrapings for microbiology studies primarily to exclude staphylococcal infection. An antinuclear antibody test can be useful if photosensitivity is prominent. A skin biopsy is useful when other diagnoses such as lupus or chronic folliculitis are being considered. ${ }^{1,8}$

\section{Approach to the patient with rosacea}

Educating the patient about rosacea as a chronic relapsing skin condition which can be controlled but does not have a traditional 'cure' is important. Warning them that flare-ups can occur even when treated properly is also useful and plays a key role in the patient's expectations and the role of therapy.

\section{General measures}

The treatment plan will be adapted to the subtype of rosacea and then realistic expectations are set and potential adverse effects discussed. This enables the patient to participate in the choice of therapy appropriate for them and consider the balance between the disease and the treatment. ${ }^{9}$

\section{Skin care}

Sun avoidance and photoprotection are an important part of management. ${ }^{10}$ Reducing skin irritability is also key. Skin care should include a gentle facial cleanser and a moisturiser or barrier repair product, as this can adjunctively improve therapeutic outcomes and reduce skin irritation in patients undergoing medical therapy. Cosmetic products, especially those with a green tinge, may help to cover erythema and may improve the patient's self-perception."

\section{Avoiding triggers}

Avoiding triggers such as extreme temperatures (hot or cold), ultraviolet radiation exposure, spicy foods, hot or alcoholic beverages, wind, exercise and stress, should be recommended to all patients. Hormonal replacement therapy can be used for menopausal flushing. ${ }^{12}$

It is important to ask the patient what medicines they are taking as some over-the-counter or prescription drugs may worsen rosacea or trigger flushing episodes. These include calcium channel blockers, sildenafil, nitrates, nicotinic acid and some vitamin B-related medications including niacin. ${ }^{2}$

\section{Specific treatments}

Treatment can be optimised according to the dominant features. ${ }^{9,13}$ Topical therapies are recommended for at least six weeks to effectively review the response. ${ }^{5,9}$ Topical corticosteroids should be avoided. ${ }^{14}$

Treatment for flushing and erythema may involve oral drugs with vasoconstriction properties including adrenergic antagonists including mirtazapine (alpha blocker), propranolol (beta blocker) or carvedilol (both alpha and beta blocker). ${ }^{2}$ These are used at low doses to avoid adverse effects such as hypotension, somnolence, fatigue and bronchospasm. They should be prescribed under specialist supervision, and careful monitoring is required.

Clonidine is an oral alpha ${ }_{2}$ agonist that has been used for flushing. However, topical alpha ${ }_{2}$ agonists are preferred because they target the skin and carry less risk of systemic adverse effects. Brimonidine is a topical alpha ${ }_{2}$ agonist which can reduce erythema for up to 12 hours through direct cutaneous vasoconstriction. Brimonidine $0.33 \%$ gel is very useful for some people when not used on a daily basis., ${ }^{6,9}$ Post-treatment rebound erythema may occur, and in general telangiectases will not clear.

\section{Erythematotelangiectatic rosacea}

Topical treatments for this form of rosacea include metronidazole, azelaic acid and brimonidine. They can be used alone or in combination. Metronidazole works as an anti-inflammatory drug by altering neutrophil chemotaxis and inactivating reactive oxygen species. Metronidazole $0.75 \%$ has been shown to reduce erythema, papules and pustules in multiple trials of patients with moderate to severe rosacea. It is usually well tolerated with minor local adverse effects such as skin irritation. ${ }^{9,15}$

Topical azelaic acid is an over-the-counter preparation which has anti-inflammatory, anti-keratinising and antibacterial effects. A $15 \%$ gel and 20\% lotion are available and can be applied once or twice daily. Adverse effects may include skin irritation, but azelaic acid is usually well tolerated and can be used for long periods of time. ${ }^{8,16}$

Laser therapy, including vascular lasers or intense pulse light, may help to reduce refractory background erythema and clinically significant telangiectases, but will not reduce the frequency of flushing episodes. Different laser therapies that target the vessels have been used such as $595 \mathrm{~nm}$ pulsed dye laser, Nd:YAG and other vascular lasers, or intense pulsed light therapy. These should be administered by an experienced and trained laser therapist and the number of sessions and length of treatment varies for each individual.11,17 


\section{Papulopustular rosacea}

Combining topical treatments with oral antibiotics may be needed for papulopustular rosacea. Topical treatments include metronidazole, azelaic acid, ivermectin and dapsone.

Ivermectin (1\% cream) is useful for mild to moderate rosacea. It has an anti-inflammatory effect as well as having an effect on Demodex mites, which may activate the local immune response to produce the pustules. It is applied once daily for up to four months, and the course may be repeated if needed. 5,15

Topical dapsone is a sulfone antibacterial with antiinflammatory actions. It was recently approved for acne in Australia, but in the USA it is approved for rosacea. Dapsone $7.5 \%$ gel is applied once daily for up to 12 weeks. It should be avoided in those with known glucose-6-phosphate dehydrogenase deficiency. ${ }^{18}$

Oral antibiotics used in papulopustular rosacea include minocycline, doxycycline, erythromycin, clarithromycin and clindamycin. Their effectiveness at sub-antimicrobial doses is mostly due to their anti-inflammatory properties rather than a direct antimicrobial mechanism. Although bacteria may contribute to this form of rosacea, evidence for this is scant. ${ }^{3}$

Doxycycline $40 \mathrm{mg}$ per day is commonly given in the USA as a sub-antimicrobial dose. The risk of resistance at this dose is less than with higher doses. In Australia 50 mg daily is used (range 25-100 mg). Photosensitivity is the main adverse effect, and sun avoidance or sunscreens may be required, especially during the summer months. Minocycline is probably a more effective agent but the increased risk of pigmentation, liver disorders and lupus-like syndrome limits its long-term use.

The goal of oral therapy is to improve the rosacea to a point where control is achieved by topical therapies. Treatment duration varies from four weeks to supress flares to many months for long-term disease suppression. With lower doses, adverse effects like headache, photosensitivity, diarrhoea and mucosal candidiasis are relatively uncommon. The antibiotics should be taken with food. 9,10,13

Erythromycin and clarithromycin are generally used in patients who are intolerant or have refractory disease to tetracyclines (e.g. doxycycline, minocycline). Topical or oral erythromycin is sometimes used in pregnant women with papulopustular rosacea.

Oral isotretinoin is usually reserved for patients who are intolerant to oral or systemic therapies. Its effect is thought to be secondary to the downregulation of the local cutaneous immunity, although an alteration in the lipid environment of the skin cannot be excluded. Low-dose isotretinoin ( $10 \mathrm{mg}$ daily) may be effective and have less adverse effects. The teratogenicity and adverse effects of isotretinoin requires routine clinical and laboratory monitoring for safety. Referral to a dermatologist is therefore recommended., ${ }^{9,19}$

\section{Phymatous rosacea}

Oral isotretinoin is also used in phymatous rosacea as with papulopustular rosacea.

The hypertrophied tissue in patients with phymatous rosacea can be reshaped and contoured with ablative lasers including carbon dioxide or electrosurgery devices. Treatment is aimed at debulking the excess tissue and then sculpting the disfigured area. Lasers produce less bleeding when compared to traditional surgery. Patients may be referred to a dermatologist or plastic surgeon for these therapies. ${ }^{11,17}$ Traditional surgery involving scalpel and loop electrosurgical excision are also used to debulk and sculpt the nose, but experience in this is required as precision may be more difficult compared to laser treatments. ${ }^{10}$

\section{Ocular rosacea}

Lid care and artificial tears are used for ocular rosacea, as are oral tetracyclines. Ciclosporin drops are reserved for moderate to severe cases and are prescribed by a consultant ophthalmologist.?

\section{Patient monitoring}

If there is an important clinical improvement in the first six weeks, the patient may need to stay on therapy for at least six months. Patients receiving oral antibiotics for six months with stable or improving rosacea should have the dose tapered as tolerated.

If the response is inadequate, therapy is completed for another six weeks and compliance should be assessed. Consider the differential diagnosis at this stage. If the diagnosis of rosacea remains, alternative regimens of oral antibiotics or switching of topical treatment may be considered. If this is not successful, consider oral isotretinoin or laser/light therapies. ${ }^{5,9}$

\section{Conclusion}

Rosacea can be a challenging condition to treat. Tailoring therapies to the type of rosacea is an important part of management.

Information about possible triggers of flushing can allow the patient to decide which are important for them. One goal should be to reduce treatment from oral to topical when possible, advise on physical therapies including laser treatment if appropriate, and to be able to explain both physical and medical management of rosacea. Asking about ocular rosacea should be considered to ensure eye health is maintained where possible. For those with severe disease or with refractory cutaneous or ocular rosacea, referral to a specialist may be required. «

Conflict of interest: none declared 
<ustralian Prescriber

\section{REFERENCES}

1. Two AM, Wu W, Gallo RL, Hata TR. Rosacea: part I. Introduction, categorization, histology, pathogenesis, and risk factors. J Am Acad Dermatol 2015;72:749-58. https://doi.org/10.1016/j.jaad.2014.08.028

2. Holmes $A D$, Steinhoff M. Integrative concepts of rosacea pathophysiology, clinical presentation and new therapeutics. Exp Dermatol 2017;26:659-67. https://doi.org/10.1111/exd.13143

3. Whitfeld M, Gunasingam N, Leow LJ, Shirato K, Preda V. Staphylococcus epidermidis: a possible role in the pustules of rosacea. J Am Acad Dermatol 2011;64:49-52. https://doi.org/10.1016/j.jaad.2009.12.036

4. Addor FA. Skin barrier in rosacea. An Bras Dermato 2016:91:59-63. https://doi.org/10.1590/abd1806-4841.20163541

5. Asai Y, Tan J, Baibergenova A, Barankin B, Cochrane CL, Humphrey S, et al. Canadian clinical practice guidelines for rosacea. J Cutan Med Surg 2016;20:432-45. https://doi.org/10.1177/1203475416650427

6. Steinhoff M, Schmelz M, Schauber J. Facial erythema of rosacea - aetiology, different pathophysiologies and treatment options. Acta Derm Venereol 2016;96:579-86. https://doi.org/10.2340/00015555-2335

7. Webster G, Schaller M. Ocular rosacea: a dermatologic perspective. J Am Acad Dermatol 2013;69(Suppl 1):S42-3 https://doi.org/10.1016/j.jaad.2013.04.040

8. Fallen RS, Gooderham M. Rosacea: update on management and emerging therapies. Skin Therapy Lett 2012;17:1-4.

9. Two AM, Wu W, Gallo RL, Hata TR. Rosacea: part II. Topical and systemic therapies in the treatment of rosacea. J Am Acad Dermatol 2015;72:761-70. https://doi.org/10.1016/j.jaad.2014.08.027

10. Del Rosso JQ, Thiboutot D, Gallo R, Webster G, Tanghetti E, Eichenfield LF, et al.; American Acne \& Rosacea Society. Consensus recommendations from the American Acne \& Rosacea Society on the management of rosacea, part 5: a guide on the management of rosacea. Cutis 2014;93:134-8.

11. Lanoue J, Goldenberg G. Therapies to improve the cosmetic symptoms of rosacea. Cutis 2015;96:19-26.
12. Sadeghian A, Rouhana H, Oswald-Stumpf B, Boh E. Etiologies and management of cutaneous flushing: nonmalignant causes. J Am Acad Dermatol 2017;77:391-402. https://doi.org/10.1016/j.jaad.2016.12.031

13. van Zuuren EJ, Fedorowicz Z, Carter B, van der Linden MM, Charland L. Interventions for rosacea. Cochrane Database Syst Rev 2015:CD003262. https://doi.org/ 10.1002/14651858.CD003262.pub5

14. Del Rosso JQ, Thiboutot D, Gallo R, Webster G, Tanghetti E, Eichenfield $\mathrm{L}$, et al. Consensus recommendations from the American Acne \& Rosacea Society on the management of rosacea, part 1: a status report on the disease state, general measures, and adjunctive skin care. Cutis 2013;92:234-40.

15. Taieb A, Khemis A, Ruzicka T, Barańska-Rybak W, Berth-Jones J, Schauber J, et al.; Ivermectin Phase III Study Group. Maintenance of remission following successful treatment of papulopustular rosacea with ivermectin $1 \%$ cream vs. metronidazole $0.75 \%$ cream: 36-week extension of the ATTRACT randomized study. J Eur Acad Dermatol Venereol 2016;30:829-36. https://doi.org/10.1111/jdv.13537

16. Chang BP, Kurian A, Barankin B. Rosacea: an update on medical therapies. Skin Therapy Lett 2014;19:1-4.

17. Tanghetti E, Del Rosso JQ, Thiboutot D, Gallo R, Webster G, Eichenfield LF, et al.; American Acne \& Rosacea Society. Consensus recommendations from the American Acne \& Rosacea Society on the management of rosacea, part 4: a status report on physical modalities and devices. Cutis 2014;93:71-6

18. Faghihi G, Khosravani P, Nilforoushzadeh MA, Hosseini SM Assaf $F$, Zeinali $N$, et al. Dapsone gel in the treatment of papulopustular rosacea: a double-blind randomized clinical trial. J Drugs Dermatol 2015;14:602-6.

19. Sbidian E, Vicaut É, Chidiack H, Anselin E, Cribier B, Dréno B, et al. A randomized-controlled trial of oral low-dose isotretinoin for difficult-to-treat papulopustular rosacea. J Invest Dermatol 2016;136:1124-9. https://doi.org/10.1016/ j.jid.2016.01.025 